\title{
The utility of magnetic resonance imaging in addition to computed tomography scans in the evaluation of cervical spine injuries: a study of obtunded blunt trauma patients
}

\author{
Bernard Puang Huh Lau ${ }^{1} \cdot$ Hwee Weng Dennis Hey ${ }^{1}$ (i) $\cdot$ Eugene Tze-Chun Lau ${ }^{1} \cdot$ \\ Pei Yi Nee ${ }^{2} \cdot$ Kimberly-Anne Tan $^{1} \cdot$ Wah Tze Tan ${ }^{3}$
}

Received: 30 January 2017 / Revised: 3 August 2017 / Accepted: 26 September 2017 / Published online: 9 October 2017

(C) Springer-Verlag GmbH Germany 2017

\begin{abstract}
Purpose Evidence guiding the use of CT and MRI scans in blunt trauma patients who are obtunded remains controversial. This study aims to determine and predict if computed tomography (CT) scans alone can be performed without risking oversight of substantial injuries found on follow-up magnetic resonance imaging (MRI).

Methods This is a retrospective cohort study of 63 blunt trauma patients with a Glasgow Coma Scale of $<8$. Data were collated from electronic medical records and included patient demographics, premorbid mobility, mechanism of injury, suspected level of injury and neurological examination findings. Patients were urgently evaluated using CT scans, followed by non-contrast MRI scans within $48 \mathrm{~h}$ of
\end{abstract}

Hwee Weng Dennis Hey

dennis_hey@nuhs.edu.sg

Bernard Puang Huh Lau

bernard_ph_lau@nuhs.edu.sg

Eugene Tze-Chun Lau

eugene_tc_lau@nuhs.edu.sg

Pei Yi Nee

pei_yi_nee@nuhs.edu.sg

Kimberly-Anne Tan

kimberlyannetan@gmail.com

Wah Tze Tan

wahze@yahoo.com

1 University Orthopaedic, Hand and Reconstructive Microsurgery Cluster (UOHC), National University Hospital, 1E Kent Ridge Road, NUHS Tower Block Level 11, Singapore 119228, Singapore

2 Division of Family Medicine, National University Hospital, Singapore, Singapore

3 Department of Anaesthesia, Ng Teng Fong General Hospital, Singapore, Singapore admission. The accuracy of CT scan was evaluated using MRI as a reference. Adjusted multivariable analysis was also performed to identify predictors for findings detected on MRI but not on CT.

Results The mean age of patients was 42.3 years and $90.5 \%$ were males. CT scans had a high specificity of $100 \%$ and sensitivity of $87.2 \%$. Predictors of MRI abnormalities include females, patients with relatively milder mechanisms of injury, patients with suspected thoracic spine injury, and CT scan findings of facet dislocation and intracranial haemorrhage. There was no predictor for spinal cord oedema.

Conclusions MRI should be performed in the presence of the aforementioned predictive factors and in the presence of neurological deficits. Otherwise, patients can be treated medically without the fear of missing a substantial cervical injury.

Keywords Cervical spine - Computed tomography . Magnetic resonance imaging - Obtunded patients .

Predictors

\section{Introduction}

The assessment for substantial injuries in the cervical spine following trauma is of high importance. Missed spinal injuries can have fatal consequences [1] or result in permanent paralysis and lifelong disability [1]. Moreover, early clearance of the cervical spine facilitates assessment of head and neck injuries [2]. If a definitive airway is subsequently required, this can also be performed efficiently without the need for neck immobilization [3].

The clearance of cervical spine from injuries is well established in patients who are conscious and alert [4]. In obtunded patients who lack reliable clinical features, 
assessment of the cervical spine is heavily reliant on imaging. Coupled with the high likelihood of multiple concomitant injuries [5] and the higher risk of desaturation requiring a definitive airway [3], the employment of computed tomography (CT) and magnetic resonance imaging (MRI) scans are common. However, there is insufficient evidence and no consensus to guide the use of these imaging modalities $[4,6]$.

Considering the benefits of early cervical spine clearance and risks of prolonged immobilization, it is, therefore, prudent to define the effectiveness and hence the role for the usage of CT and MRI scan, or both, in the assessment of cervical spine injuries for obtunded patients. In this study, we reviewed a large cohort of obtunded patients presented to a level one trauma center after blunt trauma over a period of 5 years and determined the effectiveness of CT scan alone and when supplemented with a subsequent MRI.

\section{Methodology}

\section{Study design}

This is a large retrospective cohort study conducted in a level 1 trauma center. The hospital provides tertiary healthcare services for all clinical subspecialties and has dedicated intensive care units supporting complex trauma cases. The division of spine surgery comprises seven fellowship-trained spine surgeons rostered to attend trauma activation daily on a $24 \mathrm{~h}$ basis. This study is approved by the ethics committee of the national domain-specific review board (DSRB-2013-00288).

\section{Patient population}

All patients seen at the Emergency Medicine Department (EMD) due to blunt trauma, defined as obtunded by the Glasgow Coma Scale (GCS) of $\leq 8$ and subsequently admitted to the intensive care unit (ICU) between 1st January 2008 and 31st December 2012 were included in the study.

We excluded patients who (1) have incomplete data due to electronic downtime during admission, (2) have been transferred from another hospital with CT or MRI scans already performed, and (3) patients who require emergency surgery following CT scan as a form of resuscitation and would not be able to perform an MRI scan due to medical reasons.

Out of 638 patients admitted to the intensive care units over this period, 66 patients met the inclusion. Three patients (4.5\%) were excluded based on the aforementioned reasons.

\section{Materials and methods}

All patient data were retrieved from the hospital electronic records by a single clinician. The information collected includes patient demographics (age, gender), premorbid mobility, mechanism of injury, suspected injury level and available neurological findings.

Based on the clinical workflow in our institution, all patients who suffered blunt traumatic injuries and are mentally obtunded are evaluated using the CT scan on an emergency basis once the initial resuscitation has been adequately performed. The CT scan is performed as a non-contrast study for the head, cervical spine, thorax, abdomen, and pelvis with $10 \mathrm{~mm}$ axial cuts. This is in accordance with the ATLS guidelines, ninth edition [7]. The purpose of this CT scan is to evaluate patients for cervical spine injuries and at the same time assess brain and visceral injuries.

All patients except those that require emergency surgery will be scheduled for an interval MRI scan of the cervical spine for clearance of injuries as part of the standard clinical workflow. The MRI scans are performed without contrast and within $48 \mathrm{~h}$ of admission after the patient condition has been stabilized. Cervical immobilization is removed only after all the CT and MRI images have been reviewed by the attending spine or trauma consultant and following confirmation of the final report issued by a senior radiologist to ensure that no substantial injuries are missed.

\section{Statistical analysis}

All information was entered into Microsoft Excel data spreadsheet (Excel 2013, Microsoft Corp., Redmond, WA, USA). Statistical analysis was performed using Statistical Package for Social Science (SPSS Inc., Chicago, IL version 16). Baseline patient characteristics and CT/MRI findings were shown. Categorical variables were presented as numbers with proportions and continuous variables as means with standard deviations.

Using MRI as the gold standard imaging [8], the results of CT scans in terms of sensitivities and specificities were computed. This also includes the sensitivities and specificities of the CT scan identifying MRI-specific diagnosis (disc rupture/herniation, posterior ligamentous complex injury, epidural haematoma, spinal cord oedema, bone oedema without fracture) via associated findings which can also be detected on CT scan. Multivariate analyses were performed using stepwise logistic regression to identify predictors for each of the findings unique to MRI. Only significant univariate covariates are included in the statistical modeling. Significance was set at $p<0.05$. 


\section{Results}

The mean age of the patients was 42.3 (SD 18.2) years. Road traffic accidents accounted for $58.7 \%$ of all polytrauma, followed by falls (36.5\%) and direct blunt force (4.8\%). Patients who could not be assessed due to fractured limb immobilization constitute $12.7 \%$ of the patients (Table 1).

Table 2 shows the findings on CT/MRI scan imaging that was performed.

CT scan found a variety of bone injuries. A total of 11 patients (17.5\%) had concomitant ossification of the posterior longitudinal ligament.

MRI which was made the gold standard modality for assessing injury to the cervical spine, was able to detect additional pathologies that would not be detected via $\mathrm{CT}$ alone. However, most patients with these conditions

Table 1 Baseline patient demographic and clinical features obtained at emergency medicine department

\begin{tabular}{|c|c|c|}
\hline Demographics/clinical features & $N=63$ & Percentage $(\%)$ \\
\hline \multicolumn{3}{|l|}{ Gender } \\
\hline Male & 57 & 90.5 \\
\hline Female & 6 & 9.5 \\
\hline \multicolumn{3}{|l|}{ Ethnicity } \\
\hline Chinese & 40 & 63.5 \\
\hline Malay & 7 & 11.1 \\
\hline Indian & 12 & 19.0 \\
\hline Others & 4 & 6.4 \\
\hline \multicolumn{3}{|l|}{ Premorbid mobility } \\
\hline No walking aid & 61 & 96.8 \\
\hline Uses walking aid & 2 & 3.2 \\
\hline \multicolumn{3}{|l|}{ Mechanism of injury } \\
\hline Fall from height & 11 & 17.5 \\
\hline Fall from standing height & 12 & 19.0 \\
\hline \multicolumn{3}{|l|}{ RTA } \\
\hline Motorcyclist & 20 & 31.7 \\
\hline Car & 10 & 15.9 \\
\hline Lorry/van & 5 & 7.9 \\
\hline Cyclist/pedestrian & 2 & 3.2 \\
\hline Direct blunt force & 3 & 4.8 \\
\hline \multicolumn{3}{|l|}{ Suspected injury level } \\
\hline Cervical spine injury & 51 & 81.0 \\
\hline Thoracic spine injury & 14 & 22.2 \\
\hline Lumbar spine injury & 3 & 4.8 \\
\hline \multicolumn{3}{|l|}{ Neurology } \\
\hline Normoreflexia & 20 & 36.4 \\
\hline Upper limb areflexia & 28 & 50.9 \\
\hline Lower limb areflexia & 34 & 61.8 \\
\hline Lax anal tone & 23 & 41.8 \\
\hline Unable to assess & 8 & 12.7 \\
\hline Total patients & 63 & 100.0 \\
\hline
\end{tabular}

Table 2 Significant findings on MRI and CT scans

\begin{tabular}{lcc}
\hline Significant findings of scans & Number & Percentage (\%) \\
\hline CT scan findings & 37 & 58.7 \\
Vertebral body fracture & 14 & 22.2 \\
Bony fragment retropulsion & 17 & 27.0 \\
Transverse process fracture & 19 & 30.2 \\
Pedicle/lamina fracture & 17 & 27.0 \\
Spinous process fracture & 10 & 15.9 \\
Articular facet fracture & 20 & 31.7 \\
Vertebral subluxation & 9 & 14.3 \\
Unilateral facet dislocation & 4 & 6.3 \\
Bilateral facet dislocation & 11 & 17.5 \\
Ossification of the posterior longitudi- & & \\
$\quad$ nal ligament (OPLL) & & \\
MRI (specific) scan findings & 12 & 19.1 \\
Disc rupture/herniation & 18 & 28.6 \\
Posterior ligamentous complex injury & 16 & 25.4 \\
Epidural haematoma & 36 & 57.1 \\
Spinal cord oedema & 7 & 11.1 \\
Bone oedema without fracture & &
\end{tabular}

have concomitant positive CT findings that would have prompted an MRI scan with the exception of seven patients $(11.1 \%)$ with bone oedema but without fracture. These patients can be assumed to have a missed injury if only a CT scan was performed without a routine MRI to follow-up. This resulted in the sensitivity of CT scan to be $87.2 \%$. Since there were no patients who had findings on CT but not on MRI, the specificity of CT scan was $100 \%$.

Multivariate analysis to identify predictors for MRIspecific findings that would not have been prompted by the CT scan are shown in Table 3. Except for spinal cord oedema which was found to have no significant clinical or CT predictors, common clinical predictors include females ( $p=0.007$, OR 6.14) and direct blunt force $(p=0.006)$ which was predictive of posterior ligamentous complex injury, fall from standing height which was predictive of epidural haematoma ( $p=0.034)$ and suspected thoracic spine injury which was predictive of bone oedema without fracture ( $p=0.011$, OR 14.64). CT scan findings of unilateral facet dislocation were predictive for bone oedema without fracture ( $p=0.019$, OR 5.25), bilateral facet dislocation was predictive of disc rupture/herniation and epidural haematoma ( $p=0.020$, OR 16.13 and $p=0.017$ and OR 10.62, respectively), and intracranial haemorrhage was predictive of epidural haematoma ( $p=0.004$, OR 10.75). 
Table 3 Significant multivariate predictors for significant findings on MRI scans not identified on CT scans

\begin{tabular}{|c|c|c|c|c|c|}
\hline Significant MRI findings & Predictive covariates & $p$ value & Odds ratio & Lower limit & Upper limit \\
\hline Disc rupture/herniation & Bilateral facet dislocation & 0.020 & 16.13 & 1.48 & 175.23 \\
\hline \multirow[t]{2}{*}{ Posterior ligamentous complex injury } & Female gender & 0.007 & 6.14 & 0.03 & 0.99 \\
\hline & Direct blunt force ${ }^{a}$ & 0.006 & - & - & - \\
\hline \multirow[t]{3}{*}{ Epidural haematoma } & Fall from standing height $\mathrm{t}^{\mathrm{b}}$ & 0.034 & 0.00 & 0.00 & 0.00 \\
\hline & Bilateral facet dislocation & 0.017 & 10.62 & 1.02 & 110.80 \\
\hline & Intracranial haemorrhage $\mathrm{e}^{\mathrm{b}}$ & 0.004 & 10.75 & 0.00 & 0.00 \\
\hline Spinal cord oedema & No significant multivariate predictor & & & & \\
\hline \multirow[t]{2}{*}{ Bone oedema without fracture } & Suspected thoracic spine injury & 0.011 & 14.64 & 2.36 & 90.85 \\
\hline & Unilateral facet dislocation & 0.019 & 5.25 & 0.94 & 29.44 \\
\hline
\end{tabular}

${ }^{a}$ Unable to provide odds ratio as there were no patients with direct blunt force in the cohort without significant findings on MRI scans

${ }^{\mathrm{b}}$ Odds ratio $=0.00$, as there were no patients who fell from standing height in the cohort with significant findings on MRI scans

\section{Discussion}

Trauma is a worldwide pandemic with the number of hospital admissions increasing over the past 10 years [6]. It is a significant economic burden to almost every society [9]. Patients who are obtunded as a result of blunt trauma poses a diagnostic challenge to the attending doctor in terms of the existence of cervical injury [5, 10]. In fact, Milby et al. found that cervical spine injury is more common in obtunded patients [11]. In these patients, the clinical features are often inadequate to provide conclusive evidence for cervical clearance. As such, established criteria such as the National Emergency X-Radiography Utilization Study (NEXUS) Low-Risk Criteria (NLC) [12] or the Canadian cervical spine rule (CCR) cannot be used [13].

Since there are numerous advantages for early detection and clearance of the cervical spine from substantial injuries such as facilitation of resuscitation [2], allowing better assessment of head/neck injuries [14], prevention of longterm immobilization complications such as pressure sores [14] and ventilator-related complications such as pneumonia [3], it is necessary to understand the capabilities of modern advanced imaging such as the CT and MRI in the acute setting for determining cervical spine injuries. This would enable timely clearance or surgical stabilization of the cervical spine [15]. No consensus today guide the use of these modalities in obtunded patients admitted to ICU with a GCS $<8$.

CT scan is now widely available in most emergency departments [16] and has been shown to be a useful modality for assessing the cervical spine in obtunded patients [17]. Large series have shown that CT scan can detect all clinically significant injuries $[10,17]$. A recent meta-analysis of ten studies involving 1850 obtunded blunt trauma patients found that a normal cervical spine CT scan is conclusive to safely rule out a significant cervical spine injury [18]. Panczykowski et al. also came to the same conclusion in their meta-analysis of 14,327 patients [19]. These studies focus more on clearing the cervical spine rather than identifying specific diagnoses that may be missed. Moreover, the greatest concern for relying on CT scan alone lies in its inability to diagnose ligamentous [20], discal [21] and neural injuries [21]. Milder forms of bone injury not surmountable to a fracture or dislocation cannot be appreciated as well [22]. While it may be debatable that these injuries are less severe and may not impact on clinical decisions, their associations with other injuries may in the authors' opinion affect management strategies. Nevertheless, the usefulness of CT scan in assessing other viscera injuries makes it a convenient and acceptable screening tool for these patients [23].

MRI which is ideal for soft tissue assessment has been regarded as the gold standard choice of investigation [22]. However, it has several limitations- (1) It cannot be done as an emergent setting in many situations requiring resuscitation [2], (2) It cannot be performed in unstable patients that require close monitoring and treatment [2], (3) It may not be available in some trauma centers [23]. Nevertheless, the presence of ligamentous, discal and neural injuries detectable only by MRI may affect clinical decisions. As such, it is still widely favored and often utilized.

Combined use of MRI and CT scan which would otherwise be considered as the ideal may at times be regarded as over-investigation $[18,19,22]$. The other disadvantages include-(1) the risks of prolonged immobilization while waiting for MRI scan in circumstances where CT may suffice, (2) the risks of aspiration incurred during MRI transfers and raised intracranial pressure during lengthy MRI scanning suggested by Dunham et al. [2], and (3) the adverse effects of prolonged cervical collar use such as raised intracranial pressure, pressure sores and pneumonia [14]. Despite the reported incidence of ligamentous injuries in a patient with normal CT scan varies from 1 to $30 \%$ [22, 24], Chiu et al. identified 14 out of 143 patients with isolated cervical ligamentous injuries admitted to their trauma unit over 
Fig. 1 A suggested algorithm to decide on the need for MRI scan after a CT scan

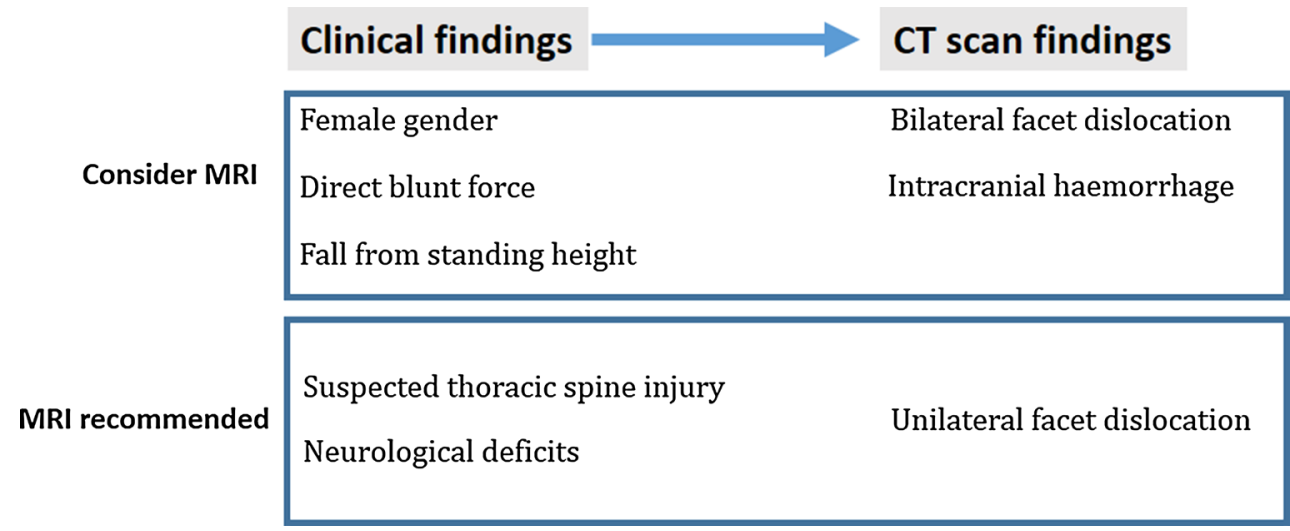

a period of 3 years [20] and concluded that ligamentous injuries without fracture of the c-spine are rare and recommended application of the practice management guidelines [6].

In our study, clinical features such as gender and seemingly milder mechanisms of injury are predisposed to having MRI findings not found on the CT scan. These findings could reflect the effectiveness of CT scan picking up most of the severe cervical spine injuries. In particular, females $(p=0.007$, OR 6.14) and direct blunt force $(p=0.006)$ was found to be predictive of posterior ligamentous complex injury, fall from standing height was predictive of epidural haematoma $(p=0.034)$ and suspected thoracic spine injury was predictive of bone oedema without fracture $(p=0.011$, OR 14.64). The presence of these features should, therefore, prompt at least an MRI scan for further workup in the future.

In terms of CT findings, unilateral facet dislocation was predictive for bone oedema without fracture ( $p=0.019$, OR 5.25) and bilateral facet dislocation was predictive of disc rupture/herniation and epidural haematoma $(p=0.020$, OR 16.13 and $p=0.017$ and OR 10.62, respectively). These findings would have prompted a follow-up MRI scan regardless and would not be as important in influencing surgical decisions ultimately. Intracranial haemorrhage, however, deserves specific mention ( $p=0.004$, OR 10.75) and should prompt further MRI evaluation of the cervical spine. No predictor was found for spinal cord oedema. This could be a result of patients having pre-existing myelomalacia from cervical myelopathy thus confounding out results. We recognize that patients who fell from standing height could also be a result, rather than a cause of cervical myelopathy [25] preventing us from drawing further conclusions. Nevertheless, it is safe to suggest that patients with neurological deficits should always be investigated with an MRI [26].

CT scan did not add any diagnosis to what was obvious on the MRI scan rendering the specificity of CT to be $100 \%$. However, eight patients with bone oedema on MRI scan would not have been detected by CT. This gave CT a sensitivity of $87.2 \%$. However, this additional information did not lead to a change in surgical plan in our cohort. Therefore, it would be reasonable to conclude that $\mathrm{CT}$ scan suffices in the evaluation of obtunded patients suffering from blunt trauma in terms of surgical management. This is supported by other studies [27]. From the aforementioned findings, a possible algorithm to decide on the need for MRI scan is shown in Fig. 1.

The limitations of this study include the possibility of interval changes between the time when CT and MRI were performed, and its retrospective nature. Although all MRI scans were performed within $48 \mathrm{~h}$ of admission after the patient condition has been stabilized, the exact time interval between the injury and MRI was unfortunately not available. This may have an impact on the MRI findings [28]. The retrospective nature of this study may also have resulted in potential biases associated with data completeness, extraction and recording. All these have to be taken into account when interpreting the results. To further strengthen the recommendations from our findings, a prospective study evaluating the combined use of CT scan and MRI should be performed.

In conclusion, $\mathrm{CT}$ scan has a high sensitivity and specificity in detecting cervical spine injuries in obtunded patients $($ GCS $<8)$ following blunt trauma. The presence of predictors for MRI-specific findings identified in this study should prompt an interval MRI scan regardless of presence or absence of neurology. In the absence of neurological deficits and the above features, patients can be treated medically without the fear of missing a substantial cervical injury.

\section{Compliance with ethical standards}

Conflict of interest The authors declare that they have no competing interests.

\section{References}

1. Marshall LF, Knowlton S, Garfin SR, Klauber MR, Eisenberg HM, Kopaniky D, Miner ME, Tabbador K, Clifton GL (1987) 
Deterioration following spinal cord injury: a multicenter study. $\mathrm{J}$ Neurosurg 66:400-404. doi:10.3171/jns.1987.66.3.0400

2. Dunham CM, Brocker BP, Collier BD, Gemmel DJ (2008) Risks associated with magnetic resonance imaging and cervical collar in comatose, blunt trauma patients with negative comprehensive cervical spine computed tomography and no apparent spinal deficit. Crit Care 12:R89. doi:10.1186/cc6957

3. Ford P, Nolan J (2002) Cervical spine injury and airway management. Curr Opin Anaesthesiol 15:193-201

4. Como JJ, Diaz JJ, Dunham CM, Chiu WC, Duane TM, Capella JM, Holevar MR, Khwaja KA, Mayglothling JA, Shapiro MB, Winston ES (2009) Practice management guidelines for identification of cervical spine injuries following trauma: update from the eastern association for the surgery of trauma practice management guidelines committee. J Trauma 67:651-659. doi:10.1097/ TA.0b013e3181ae583b

5. Halpern CH, Milby AH, Guo W, Schuster JM, Gracias VH, Stein SC (2010) Clearance of the cervical spine in clinically unevaluable trauma patients. Spine 35:1721-1728

6. Patel MB, Humble SS, Cullinane DC, Day MA, Jawa RS, Devin CJ, Delozier MS, Smith LM, Smith MA, Capella JM, Long AM, Cheng JS, Leath TC, Falck-Ytter Y, Haut ER, Como JJ (2015) Cervical spine collar clearance in the obtunded adult blunt trauma patient: a systematic review and practice management guideline from the Eastern Association for the Surgery of Trauma. J Trauma Acute Care Surg 78:430-441. doi:10.1097/ TA.0000000000000503

7. Subcommittee A, American College of Surgeons' Committee on T, International Awg (2013) Advanced trauma life support (ATLS(R)): the ninth edition. J Trauma Acute Care Surg 74:13631366. doi:10.1097/TA.0b013e31828b82f5

8. Fisher BM, Cowles S, Matulich JR, Evanson BG, Vega D, Dissanaike $S$ (2013) Is magnetic resonance imaging in addition to a computed tomographic scan necessary to identify clinically significant cervical spine injuries in obtunded blunt trauma patients? Am J Surg 206:987-993. doi:10.1016/j.amjsurg.2013.08.021 (discussion 984-993)

9. Badhiwala JH, Lai CK, Alhazzani W, Farrokhyar F, Nassiri F, Meade M, Mansouri A, Sne N, Aref M, Murty N, Witiw C, Singh S, Yarascavitch B, Reddy K, Almenawer SA (2015) Cervical spine clearance in obtunded patients after blunt traumatic injury: a systematic review. Ann Intern Med 162:429-437. doi:10.7326/ M14-2351

10. Harris TJ, Blackmore CC, Mirza SK, Jurkovich GJ (2008) Clearing the cervical spine in obtunded patients. Spine 33:1547-1553. doi:10.1097/BRS.0b013e31817926c1

11. Milby AH, Halpern CH, Guo W, Stein SC (2008) Prevalence of cervical spinal injury in trauma. Neurosurg Focus 25:E10. doi:10.3171/FOC.2008.25.11.E10

12. Tran J, Jeanmonod D, Agresti D, Hamden K, Jeanmonod RK (2016) Prospective validation of modified NEXUS cervical spine injury criteria in low-risk elderly fall patients. West J Emerg Med 17:252-257. doi:10.5811/westjem.2016.3.29702

13. Hall FM (2004) The Canadian C-spine rule. N Engl J Med 350:1467-1469 (author reply 1467-1469)

14. Hauswald M, Hsu M, Stockoff C (2000) Maximizing comfort and minimizing ischemia: a comparison of four methods of spinal immobilization. Prehosp Emerg Care 4:250-252

15. Pekmezci M, Theologis AA, Dionisio R, Mackersie R, McClellan RT (2015) Cervical spine clearance protocols in Level I, II, and III trauma centers in California. Spine J 15:398-404. doi:10.1016/j. spinee.2014.12.142
16. Berdahl CT, Vermeulen MJ, Larson DB, Schull MJ (2013) Emergency department computed tomography utilization in the United States and Canada. Ann Emerg Med 62(486-494):e483. doi:10.1016/j.annemergmed.2013.02.018

17. Hogan GJ, Mirvis SE, Shanmuganathan K, Scalea TM (2005) Exclusion of unstable cervical spine injury in obtunded patients with blunt trauma: is MR imaging needed when multi-detector row CT findings are normal? Radiology 237:106-113. doi:10.1148/radiol.2371040697

18. Raza M, Elkhodair S, Zaheer A, Yousaf S (2013) Safe cervical spine clearance in adult obtunded blunt trauma patients on the basis of a normal multidetector CT scan - a meta-analysis and cohort study. Injury 44:1589-1595. doi:10.1016/j. injury.2013.06.005

19. Panczykowski DM, Tomycz ND, Okonkwo DO (2011) Comparative effectiveness of using computed tomography alone to exclude cervical spine injuries in obtunded or intubated patients: meta-analysis of 14,327 patients with blunt trauma. J Neurosurg 115:541-549. doi:10.3171/2011.4.JNS101672

20. Chiu WC, Haan JM, Cushing BM, Kramer ME, Scalea TM (2001) Ligamentous injuries of the cervical spine in unreliable blunt trauma patients: incidence, evaluation, and outcome. J Trauma 50:457-463 (discussion 464)

21. Maung AA, Johnson DC, Barre K, Peponis T, Mesar T, Velmahos GC, McGrail D, Kasotakis G, Gross RI, Rosenblatt MS, Sihler KC, Winchell RJ, Cholewczynski W, Butler KL, Odom SR, Davis KA, Group RMCSS (2016) Cervical spine MRI in patients with negative CT: a prospective, multicenter study of the Research Consortium of New England Centers for Trauma (ReCONECT). J Trauma Acute Care Surg. doi:10.1097/TA.0000000000001322

22. Menaker J, Philp A, Boswell S, Scalea TM (2008) Computed tomography alone for cervical spine clearance in the unreliable patient-are we there yet? J Trauma 64:898-903. doi:10.1097/ TA.0b013e 3181674675 (discussion 894-903)

23. Shah LM, Flanders AE (2017) Update on new imaging techniques for trauma. Neurosurg Clin N Am 28:1-21. doi:10.1016/j. nec.2016.08.006

24. Stassen NA, Williams VA, Gestring ML, Cheng JD, Bankey PE (2006) Magnetic resonance imaging in combination with helical computed tomography provides a safe and efficient method of cervical spine clearance in the obtunded trauma patient. J Trauma 60:171-177. doi:10.1097/01.ta.0000197647.44202.de

25. Kimura A, Seichi A, Takeshita K, Inoue H, Kato T, Yoshii T, Furuya T, Koda M, Takeuchi K, Matsunaga S, Seki S, Ishikawa Y, Imagama S, Yamazaki M, Mori K, Kawasaki Y, Fujita K, Endo K, Sato K, Okawa A (2016) Fall-related deterioration of subjective symptoms in patients with cervical myelopathy. Spine. doi:10.1097/BRS.0000000000001798

26. Martinez-Perez R, Munarriz PM, Paredes I, Cotrina J, Lagares A (2017) Cervical spinal cord injury without computed tomography evidence of trauma in adults: magnetic resonance imaging prognostic factors. World Neurosurg 99:192-199. doi:10.1016/j. wneu.2016.12.005 (Epub 2016 Dec 12)

27. Malhotra A, Wu X, Kalra VB et al (2017) Utility of MRI for cervical spine clearance after blunt traumatic injury: a meta-analysis. Eur Radiol (Germany) 27(3):1148-1160

28. Rutges JPHJ, Kwon BK, Heran M, Ailon T, Street JT, Dvorak MF (2017) A prospective serial MRI study following acute traumatic cervical spinal cord injury. Eur Spine J. doi:10.1007/s00586-0175097-4 (Epub ahead of print) 